\title{
openheart Speckle tracking strain and ECG heterogeneity correlate in transcatheter aortic valve replacement-induced left bundle branch blocks and right ventricular paced rhythms
}

\author{
Gordon M Burke (D) , ${ }^{1,2}$ Bruna Araujo Silva, ${ }^{3}$ Alexandre A Marum, ${ }^{3}$ \\ Alexandre L Bortolotto, ${ }^{3}$ Bruce D Nearing, ${ }^{2,3}$ Michael J Chen, ${ }^{3}$ Sarah Fostello, ${ }^{3}$ \\ Jeffrey J Popma, ${ }^{2,3}$ Richard L Verrier, ${ }^{2,3}$ James D Chang ${ }^{2,3}$
}

To cite: Burke GM,

Araujo Silva B, Marum AA, et al. Speckle tracking strain and ECG heterogeneity correlate in transcatheter aortic valve replacement-induced left bundle branch blocks and right ventricular paced rhythms. Open Heart 2021;8:e001542.

doi:10.1136/

openhrt-2020-001542

Received 29 July 2021 Accepted 30 August 2021
Check for updates

(C) Author(s) (or their employer(s)) 2021. Re-use permitted under CC BY-NC. No commercial re-use. See rights and permissions. Published by BMJ.

${ }^{1}$ Cardiology, VA Boston Healthcare System, West Roxbury, Massachusetts, USA ${ }^{2}$ Harvard Medical School, Boston, Massachusetts, USA ${ }^{3}$ Cardiology, Beth Israel Deaconess Medical Center, Boston, Massachusetts, USA

Correspondence to Dr Gordon M Burke; Gordon. Burke@va.gov

\section{ABSTRACT}

Objective Transcatheter aortic valve replacement (TAVR) complications include left bundle branch block (LBBB) and right ventricular paced rhythm (RVP). We hypothesised that changes in electrocardiographic heterogeneity would correlate better with speckle tracking strain measures than with left ventricular ejection fraction (LVEF) on transthoracic echocardiogram (TTE) among patients with TAVR-induced conduction abnormalities.

Methods We reviewed medical records of 446 consecutive patients who underwent TAVR at our institution. Of the 238 patients with 12-lead electrocardiograms (ECGs) that met our inclusion criteria, 58 had pre-TAVR and post-TAVR TTEs adequate for strain assessment. We compared patients who did not have an LBBB or RVP pre-TAVR and post-TAVR (controls, $n=11$ ) with patients who developed LBBBs $(n=11)$ and who required RVPs $(n=10)$ post-TAVR. In our study population ( $n=32,41 \%$ female, mean age 85.8 years), we evaluated QRS complex duration, R-wave heterogeneity (RWH), Twave heterogeneity (TWH), LVEF, global longitudinal strain (GLS) and mechanical dispersion (MD).

Results TAVR-induced changes on ECG did not correlate with LVEF. TAVR-induced changes in MD and QRS complex duration correlated among all patients $(r=0.4, p=0.04)$. GLS and RWH correlated among RVP patients $(r=0.7$, $\mathrm{p}=0.00003)$. MD and TWH correlated among LBBB patients $(r=0.7, p=0.00004)$.

Conclusions In this convenience sample of patients with TAVR-induced conduction abnormalities, RWH and TWH correlated with strain measures but not with LVEF. Strain measures, RWH and TWH may offer additional insights for pre-TAVR evaluation and post-TAVR clinical management.

\section{INTRODUCTION}

Despite favourable major outcomes of transcatheter aortic valve replacement (TAVR) compared with surgical aortic valve replacement, certain complications are higher among TAVR patients, including new onset

\section{Key questions}

What is already known about this subject?

- Left bundle branch block (LBBB) and heart block requiring right ventricular paced rhythm (RVP) are the most common complications associated with transcatheter aortic valve replacement (TAVR) TAVR-induced LBBBs and RVPs have been associated with worse clinical outcomes. Current clinical guidelines for management of TAVR-induced LBBBs and RVPs highlight the lack of evidence supporting surveillance and treatment particularly regarding subsequent implantation of or upgrade to cardiac resynchronisation therapy and/or implantation of cardioverter defibrillators.

- Speckle tracking strain on transthoracic echocardiogram and R-wave and T-wave heterogeneity (RWH TWH) on ECG have been shown to be better predictors of ventricular arrhythmias, sudden cardiac death and response to cardiac resynchronisation therapy than left ventricular ejection fraction (LVEF).

What does this study add?

- This study demonstrated that speckle tracking strain, RWH and TWH more sensitively identified changes than LVEF and demonstrated that changes in RWH and TWH correlated with strain measures but not with LVEF among patients with TAVRinduced LBBBs and RVPs.

How might this impact on clinical practice?

- Further study using strain, RWH and TWH may offer additional insight to assist with surveillance and clinical management regarding cardiac resynchronisation therapy and cardioverter defibrillator implantation among patients with TAVR-induced LBBBs and RVPs over current conventional assessments, such as LVEF.

left bundle branch block (LBBB) and new onset right ventricular paced rhythm (RVP). ${ }^{1}{ }^{2}$ Studies evaluating patients with 
TAVR-induced LBBBs and RVPs have yielded varying results but have shown a trend towards a lack of recovery of left ventricular ejection fraction (LVEF) or reduction in LVEF, increased rates of heart failure rehospitalisation and increased mortality. ${ }^{3}$ The physiology associated with LBBBs and RVPs depends on the underlying aetiology of the conduction disturbance. ${ }^{45}$ Therefore, the physiology associated with TAVR-induced LBBBs and RVPs is likely unique compared with other aetiologies of these conduction abnormalities. Current clinical guidelines on TAVRinduced conduction abnormalities highlight the lack of conclusive data regarding appropriate surveillance and treatment for these patients. ${ }^{6}$ As the volume of TAVRs continues to grow, it will be increasingly important to use optimal testing modalities to evaluate which patients are at higher risk for procedure-related conduction abnormalities and to develop guidance in decision-making regarding appropriate surveillance and treatment of these complications.

Speckle tracking strain on transthoracic echocardiogram (TTE) and R-wave and T-wave heterogeneity (RWH, TWH) on electrocardiogram (ECG) have been shown to predict adverse clinical outcomes independent of LVEF. ${ }^{7-10}$ We hypothesised that RWH and TWH would correlate more closely with strain measures than with LVEF among patients with TAVR-induced conduction abnormalities.

\section{METHODS}

\section{Study population}

We retrospectively reviewed medical records of all 446 patients who underwent TAVR from 2012 to 2016 at our institution. We reviewed available digital ECGs performed at our medical centre pre-TAVR and 1-month post-TAVR. We excluded 26 patients who did not have a pre-TAVR digital ECG and 79 patients who did not have a post-TAVR digital ECG performed at our medical centre. Also, we excluded 51 patients with pre-TAVR LBBBs and 52 patients with pre-TAVR RVPs. The control group included patients who did not have an LBBB or RVP preTAVR or 1-month post-TAVR $(n=146)$. The LBBB group included patients who developed a new LBBB at the time of TAVR that was present on ECG 1-month post-TAVR $(n=48)$. The RVP group included patients requiring a new RVP at the time of TAVR that was present on ECG 1-month post-TAVR $(n=44)$. Next, we screened TTEs of these patients for the technical requirements for speckle tracking strain analysis (control group, $n=37$; LBBB group, $\mathrm{n}=11$; RVP group, $\mathrm{n}=10$ ). We randomly selected 11 patients from the control group to balance the sample size among the three groups.

\section{Electrocardiographic assessment}

12-lead ECGs were recorded pre-TAVR and 1-month post-TAVR using Cardiolab GE Prucka (GE Healthcare, Chicago, Illinois, USA) with standard filtering (0.05$150 \mathrm{~Hz}$ ), a sampling rate of 1000 samples per channel, a 16-bit A/D converter and a least significant bit resolution of $1.5 \mu \mathrm{V}$. A single physician reviewed the ECGs. The diagnosis of LBBB or RVP was made based on current recommendations. ${ }^{11}$ We assessed RWH and TWH on ECG using second central moment analysis, which quantifies the variation about the mean $\mathrm{R}$-wave and T-wave morphologies in adjoining precordial leads (in this study, V4, V5 and V6) in microvolts.

\section{Echocardiographic assessment}

TTEs were recorded pre-TAVR and 1-month post-TAVR using GE echocardiographs (GE Healthcare). A single physician, who was blinded to patient groups, analysed TTEs offline using GE software (GE Healthcare). LVEF was assessed using Simpson's biplane method. Longitudinal speckle tracking strain analyses were performed using current recommendations including three apical views of sufficient image quality (ie, adequate myocardial visualisation, no more than one segment excluded per view and recorded with at least 40 frames per second). ${ }^{12}$ Global longitudinal strain (GLS) was calculated as the average of all the peak longitudinal strain values from an 18-segment model. Time to peak longitudinal strain was measured to allow for assessment of mechanical dispersion (MD). MD was defined as the standard deviation (SD) of all the time to peak longitudinal strain measures from an 18-segment model.

\section{Statistical analysis}

Descriptive statistics of our sample population are expressed as means and SD. We compared ECG and TTE measures pre-TAVR and post-TAVR using paired Student's t-tests. We used correlation coefficients to assess correlation of change in ECG and TTE measures. Statistical analyses were performed using SAS V.9.2 (SAS Institute).

\section{Patient and public involvement statement}

There was neither patient nor public involvement in the design of the study, recruitment to the study, conduct of the study or dissemination of the study results.

\section{RESULTS}

\section{Patient and procedural characteristics}

Our study included 32 patients who met the inclusion criteria as previously described. Baseline clinical characteristics are presented in table 1 . The control group had a female predominance $(73 \%)$ compared with the other groups (LBBB 36\%, $\mathrm{p}=0.05$; RVP 10\%, $\mathrm{p}=0.001$ ). History of coronary artery disease was more prevalent in the LBBB group (91\%) compared with controls $(36 \%$, $\mathrm{p}=0.003)$. Procedural characteristics are presented in table 2. Average TAVR size was smaller in the control group $(25.4 \pm 2.4 \mathrm{~mm})$ compared with the RVP group (28.2 $\pm 2.5 \mathrm{~mm}, \mathrm{p}=0.008)$.

\section{Electrocardiographic results}

Pre-TAVR and 1-month post-TAVR ECG measures are shown in table 3 . In the control group, pre-TAVR and 


\begin{tabular}{|c|c|c|c|c|c|c|}
\hline & $\begin{array}{l}\text { All patients } \\
(n=32)\end{array}$ & $\begin{array}{l}\text { Control } \\
(n=11)\end{array}$ & $\begin{array}{l}\text { LBBB } \\
(n=11)\end{array}$ & $\begin{array}{l}\text { P value compared } \\
\text { with control }\end{array}$ & $\begin{array}{l}\text { RVP } \\
(n=10)\end{array}$ & $\begin{array}{l}\text { P value compared } \\
\text { with control }\end{array}$ \\
\hline \multicolumn{7}{|l|}{ Baseline clinical characteristics } \\
\hline Age, years & $85.8 \pm 8.9$ & $85.7 \pm 11.3$ & $85.7 \pm 4.3$ & 0.5 & $86.0 \pm 11.0$ & 0.5 \\
\hline Sex, female (\%) & 41 & 73 & 36 & 0.05 & 10 & 0.001 \\
\hline BMI $\left(\mathrm{kg} / \mathrm{m}^{2}\right)$ & $24.9 \pm 3.1$ & $24.7 \pm 2.5$ & $25.4 \pm 3.4$ & 0.3 & $24.6 \pm 3.7$ & 0.4 \\
\hline NYHA class & $2.8 \pm 0.5$ & $2.8 \pm 0.4$ & $2.8 \pm 0.8$ & 0.4 & $2.8 \pm 0.4$ & 0.4 \\
\hline STS-PROM score (\%) & $7.6 \pm 3.7$ & $6.7 \pm 4.2$ & $7.4 \pm 3.3$ & 0.4 & $8.8 \pm 3.8$ & 0.1 \\
\hline $\begin{array}{l}\text { Mean five metre walk test } \\
\text { (seconds) }\end{array}$ & $9.5 \pm 7.9$ & $7.2 \pm 3.6$ & $11.7 \pm 12.3$ & 0.2 & $8.9 \pm 4.3$ & 0.2 \\
\hline Creatinine (mg/dL) & 1.3 & 1.2 & 1.1 & 0.4 & 1.6 & 0.08 \\
\hline Atrial fibrillation (\%) & 28 & 27 & 18 & 0.3 & 40 & 0.4 \\
\hline Hypertension & 69 & 82 & 64 & 0.2 & 60 & 0.1 \\
\hline Diabetes mellitus & 28 & 18 & 36 & 0.2 & 30 & 0.3 \\
\hline Smoker within 1 year & 6 & 0 & 9 & 0.2 & 10 & 0.2 \\
\hline CAD (\%) & 66 & 36 & 91 & 0.003 & 70 & 0.07 \\
\hline Prior Ml (\%) & 16 & 0 & 27 & 0.03 & 20 & 0.07 \\
\hline Prior PCl (\%) & 28 & 27 & 36 & 0.3 & 20 & 0.4 \\
\hline Prior CABG (\%) & 22 & 9 & 27 & 0.1 & 30 & 0.1 \\
\hline \multicolumn{7}{|l|}{ Medications } \\
\hline Aspirin & 100 & 100 & 100 & 0.5 & 100 & 0.5 \\
\hline ACE inhibitor & 38 & 36 & 27 & 0.3 & 50 & 0.3 \\
\hline Beta blocker & 56 & 55 & 73 & 0.2 & 40 & 0.3 \\
\hline Statin & 69 & 64 & 82 & 0.2 & 60 & 0.4 \\
\hline \multicolumn{7}{|c|}{ 1-month post-TAVR clinical characteristics } \\
\hline \multicolumn{5}{|c|}{ Ventricular pacing frequency (\%) } & \multicolumn{2}{|l|}{$97(n=9)$} \\
\hline
\end{tabular}

BMI, body mass index; CABG, coronary artery bypass graft; CAD, coronary artery disease; LBBB, left bundle branch block; MI, myocardial infarction; NYHA, New York Heart Association; PCI, percutaneous coronary intervention; RVP, right ventricular paced rhythm; STS-PROM, Society of Thoracic Surgeons-predicted risk of mortality; TAVR, transcatheter aortic valve replacement.

post-TAVR QRS complex duration, RWH and TWH remained stable $(\mathrm{p}=0.3)$. Among the RVP group, all preTAVR ECG measures increased post-TAVR (QRS complex duration: pre-TAVR $116.2 \pm 7 \mathrm{~ms}$, post-TAVR $161.8 \pm 5 \mathrm{~ms}$, $\mathrm{p}=0.0002 ;$ RWH: pre-TAVR $331.9 \pm 33 \mu \mathrm{V}$, post-TAVR
$494.8 \pm 66 \mu \mathrm{V}, \mathrm{p}=0.01$; TWH: pre-TAVR $161.8 \pm 28 \mu \mathrm{V}$, postTAVR $337.5 \pm 40 \mu V, p=0.001$ ). Only QRS complex duration and TWH increased post-TAVR compared with preTAVR in the LBBB group (QRS complex duration: preTAVR $101.3 \pm 6 \mathrm{~ms}$, post-TAVR $146.9 \pm 6 \mathrm{~ms}, \mathrm{p}=0.000001$;

Table 2 Procedural characteristics

\begin{tabular}{lllllll}
\hline & All patients & Control & LBBB & $\begin{array}{l}\text { P value compared with } \\
\text { control }\end{array}$ & RVP & $\begin{array}{l}\text { P value compared with } \\
\text { control }\end{array}$ \\
\hline TAVR size $(\mathrm{mm})$ & $26.9 \pm 2.8$ & $25.4 \pm 2.4$ & $27.3 \pm 3.0$ & 0.06 & $28.2 \pm 2.5$ & 0.008 \\
\hline $\begin{array}{l}\text { TAVR manufacturer (\%) } \\
\quad\end{array}$ & & & & & \\
$\quad$ Medtronic & 63 & 55 & 73 & 0.2 & 60 & 0.4 \\
\hline Edwards & 31 & 45 & 18 & 0.1 & 30 & 0.2 \\
$\quad$ Boston Scientific & 6 & 0 & 9 & 0.2 & 10 & 0.2 \\
Vascular access site (\%) & & & & & & \\
$\quad$ Transfemoral & 97 & 91 & 100 & 0.2 & 100 & 0.2 \\
\hline Transaortic & 3 & 9 & 0 & 0.2 & 0 & 0.2 \\
\hline
\end{tabular}

LBBB, left bundle branch block; RVP, right ventricular paced rhythm; TAVR, transcatheter aortic valve replacement. 
Table 3 ECGs pre-TAVR and post-TAVR

\begin{tabular}{lllll}
\hline & All patients & Control & LBBB & RVP \\
\hline Heart rate pre-TAVR $(\mathrm{bpm})$ & $72.4 \pm 4$ & $77.2 \pm 9$ & $72.2 \pm 5$ & $67.5 \pm 4$ \\
\hline Heart rate post-TAVR $(\mathrm{bpm})$ & $72.6 \pm 3$ & $68.7 \pm 4.2$ & $75.2 \pm 7$ & $73.9 \pm 4$ \\
\hline P value pre-TAVR vs post-TAVR & 0.9 & 0.3 & 0.7 & 0.009 \\
\hline QRS complex duration pre-TAVR $(\mathrm{ms})$ & $104.6 \pm 3$ & $97.4 \pm 3$ & $101.3 \pm 6$ & $116.2 \pm 7$ \\
\hline QRS complex duration post-TAVR $(\mathrm{ms})$ & $134.6 \pm 5$ & $97.6 \pm 3$ & $146.9 \pm 6$ & $161.8 \pm 5$ \\
\hline P value pre-TAVR vs post-TAVR & 0.0000007 & 0.9 & 0.000001 & 0.0002 \\
\hline RWH pre-TAVR $(\mu \mathrm{V})$ & $338.0 \pm 24$ & $298.4 \pm 48$ & $384.0 \pm 39$ & $331.9 \pm 33$ \\
\hline RWH post-TAVR $(\mu \mathrm{V})$ & $407.8 \pm 33$ & $307.8 \pm 51$ & $428.8 \pm 45$ & $494.8 \pm 66$ \\
\hline P value pre-TAVR vs post-TAVR & 0.05 & 0.9 & 0.4 & 0.01 \\
\hline TWH pre-TAVR $(\mu \mathrm{V})$ & $143.0 \pm 13$ & $104.4 \pm 18$ & $164.4 \pm 18$ & $161.8 \pm 28$ \\
\hline TWH post-TAVR $(\mu \mathrm{V})$ & $256.2 \pm 31$ & $108.7 \pm 21$ & $329.7 \pm 60$ & $337.5 \pm 40$ \\
\hline P value pre-TAVR vs post-TAVR & 0.0002 & 0.8 & 0.01 & 0.001 \\
\hline
\end{tabular}

LBBB, left bundle branch block; RVP, right ventricular paced rhythm; RWH, R-wave heterogeneity; TAVR, transcatheter aortic valve replacement; TWH, Twave heterogeneity.

TWH: pre-TAVR $164.4 \pm 18 \mu \mathrm{V}$, post-TAVR $329.7 \pm 60 \mu \mathrm{V}$, $\mathrm{p}=0.01)$.

\section{Echocardiographic results}

Pre-TAVR and 1-month post-TAVR TTE measures are shown in table 4. Mean LVEF, GLS and MD post-TAVR compared with pre-TAVR demonstrated a trend towards improvement in the control group and remained stable in the RVP group $(p \geq 0.3)$. In the LBBB group, LVEF and GLS demonstrated a trend of decline in systolic function ( $\mathrm{p} \geq 0.3)$, and MD increased from pre-TAVR to post-TAVR (pre-TAVR MD $45.8 \pm 12.0 \mathrm{~ms}$, post-TAVR MD $78.5 \pm 35.1 \mathrm{~ms}, \mathrm{p}=0.02)$.

Polar maps demonstrating GLS pre-TAVR and postTAVR in a patient from each group are shown in figure 1. Polar maps from a control patient (figure 1, left column) show a pre-TAVR GLS of $-14.6 \%$ (top row) and a postTAVR GLS of $-22.0 \%$ (bottom row). Polar maps from an LBBB patient (figure 1, centre column) show a pre-TAVR GLS of $-22.3 \%$ (top row) and a post-TAVR GLS of $-1.3 \%$ (bottom row). Polar maps from an RVP patient (figure 1, right column) show a pre-TAVR GLS of $-15.5 \%$ (top row) and a post-TAVR GLS of $-16.0 \%$ (bottom row).

Polar maps demonstrating time to peak longitudinal strain pre-TAVR and 1-month post-TAVR in a patient from each group are shown in figure 2. Polar maps from a control patient (figure 2, left column) show a preTAVR MD of $79.0 \mathrm{~ms}$ (top row) and a post-TAVR MD of $49.3 \mathrm{~ms}$ (bottom row). Polar maps from an LBBB patient (figure 2, centre column) show a pre-TAVR MD of $43.5 \mathrm{~ms}$ (top row) and a post-TAVR MD of $158.5 \mathrm{~ms}$ (bottom row). Polar maps from an RVP patient (figure 2, right column) show a pre-TAVR MD of $51.9 \mathrm{~ms}$ (top row) and a postTAVR MD of $84.9 \mathrm{~ms}$ (bottom row).

\section{Correlation of electrocardiographic and echocardiographic results}

The correlation of change in TTE and ECG measures pre-TAVR and post-TAVR are shown in table 5. Among all patients, change in LVEF showed a trend towards

Table 4 Echocardiograms pre-TAVR and post-TAVR

\begin{tabular}{lllll}
\hline & All patients & Control & LBBB & RVP \\
\hline Pre-TAVR LVEF (\%) & $55.9 \pm 10.3$ & $56.3 \pm 12.6$ & $55.5 \pm 9.5$ & $56.0 \pm 8.3$ \\
\hline Post-TAVR LVEF (\%) & $54.3 \pm 11.8$ & $58.1 \pm 6.5$ & $51.1 \pm 16.7$ & $53.5 \pm 9.7$ \\
\hline P value pre-TAVR vs post-TAVR & 0.4 & 0.7 & 0.4 & 0.4 \\
\hline Pre-TAVR GLS (\%) & $-16.6 \pm 4.8$ & $-16.9 \pm 3.9$ & $-16.7 \pm 5.6$ & $-14.4 \pm 6.0$ \\
\hline Post-TAVR GLS (\%) & $-16.0 \pm 5.1$ & $-18.2 \pm 3.0$ & $-14.5 \pm 6.5$ & $-15.5 \pm 4.7$ \\
\hline P value pre-TAVR vs post-TAVR & 0.6 & 0.3 & 0.3 & 0.7 \\
\hline Pre-TAVR MD (ms) & $55.6 \pm 21.0$ & $58.6 \pm 11.7$ & $45.8 \pm 12.0$ & $63.3 \pm 31.9$ \\
\hline Post-TAVR MD (ms) & $64.6 \pm 25.3$ & $54.3 \pm 17.8$ & $78.5 \pm 35.1$ & $64.6 \pm 20.7$ \\
\hline P value pre-TAVR vs post-TAVR & 0.09 & 0.4 & 0.02 & 0.9 \\
\hline
\end{tabular}

GLS, global longitudinal strain; LBBB, left bundle branch block; LVEF, left ventricular ejection fraction; MD, mechanical dispersion; RVP, right ventricular paced rhythm; TAVR, transcatheter aortic valve replacement. 


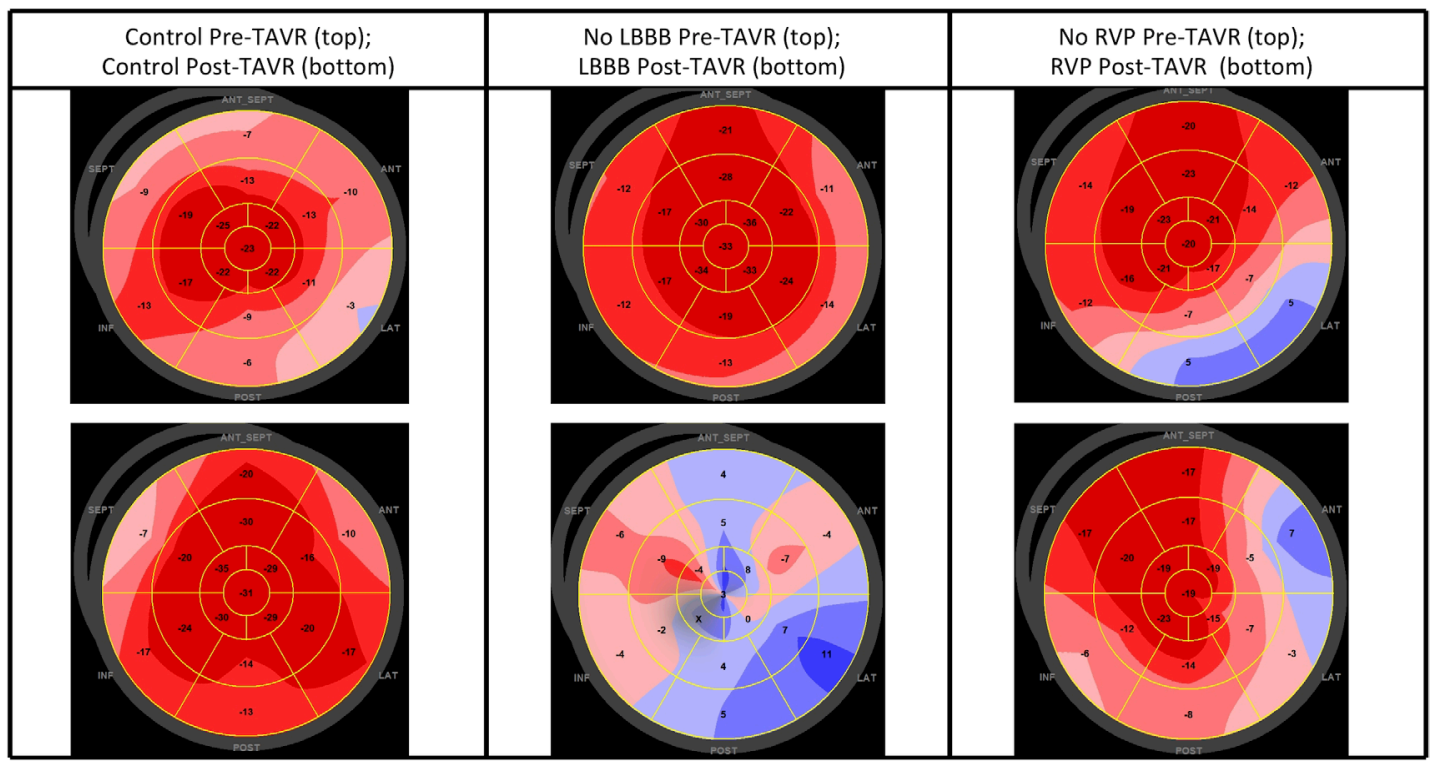

Figure 1 Pre-TAVR and post-TAVR polar maps of global longitudinal strain. Pre-TAVR (top row) and post-TAVR (bottom row) polar maps of global longitudinal strain in representative patients from each group. Left column: a control patient; centre column: a patient who developed LBBB post-TAVR; right column: a patient who required RVP post-TAVR. LBBB, left bundle branch block; RVP, right ventricular paced rhythm; TAVR, transcatheter aortic valve replacement.

negative correlation with $\mathrm{QRS}$ duration $(\mathrm{r}=-0.3, \mathrm{p}=0.1)$, RWH ( $\mathrm{r}=-0.2, \mathrm{p}=0.2)$ and TWH $(\mathrm{r}=-0.3$, $\mathrm{p}=0.07)$. In the RVP group, change in GLS and RWH strongly correlated $(\mathrm{r}=0.7, \mathrm{p}=0.00003)$. Among all patients, change in MD moderately correlated with QRS duration $(\mathrm{r}=0.4, \mathrm{p}=0.04)$ and mildly correlated with RWH ( $\mathrm{r}=0.1, \mathrm{p}=0.01)$. In the LBBB group, change in MD moderately correlated with RWH $(\mathrm{r}=0.4, \mathrm{p}=0.01)$ and strongly correlated with TWH ( $r=0.7, p=0.00004)$. In the RVP group, change in MD showed moderate negative correlation with TWH $(\mathrm{r}=-0.4, \mathrm{p}=0.03)$.
Comparisons of TWH and MD pre-TAVR and post-TAVR stratified by group are shown in figure 3. TWH $(p=0.8)$ and MD $(p=0.4)$ in the control group did not change significantly. TWH increased in the LBBB $(p=0.01)$ and RVP ( $p=0.001)$ groups. MD increased in the LBBB group $(p=0.01)$ but not in the RVP group $(p=0.8)$.

\section{DISCUSSION}

Our study identified differences pre-TAVR and postTAVR using RWH, TWH and MD that were not detected

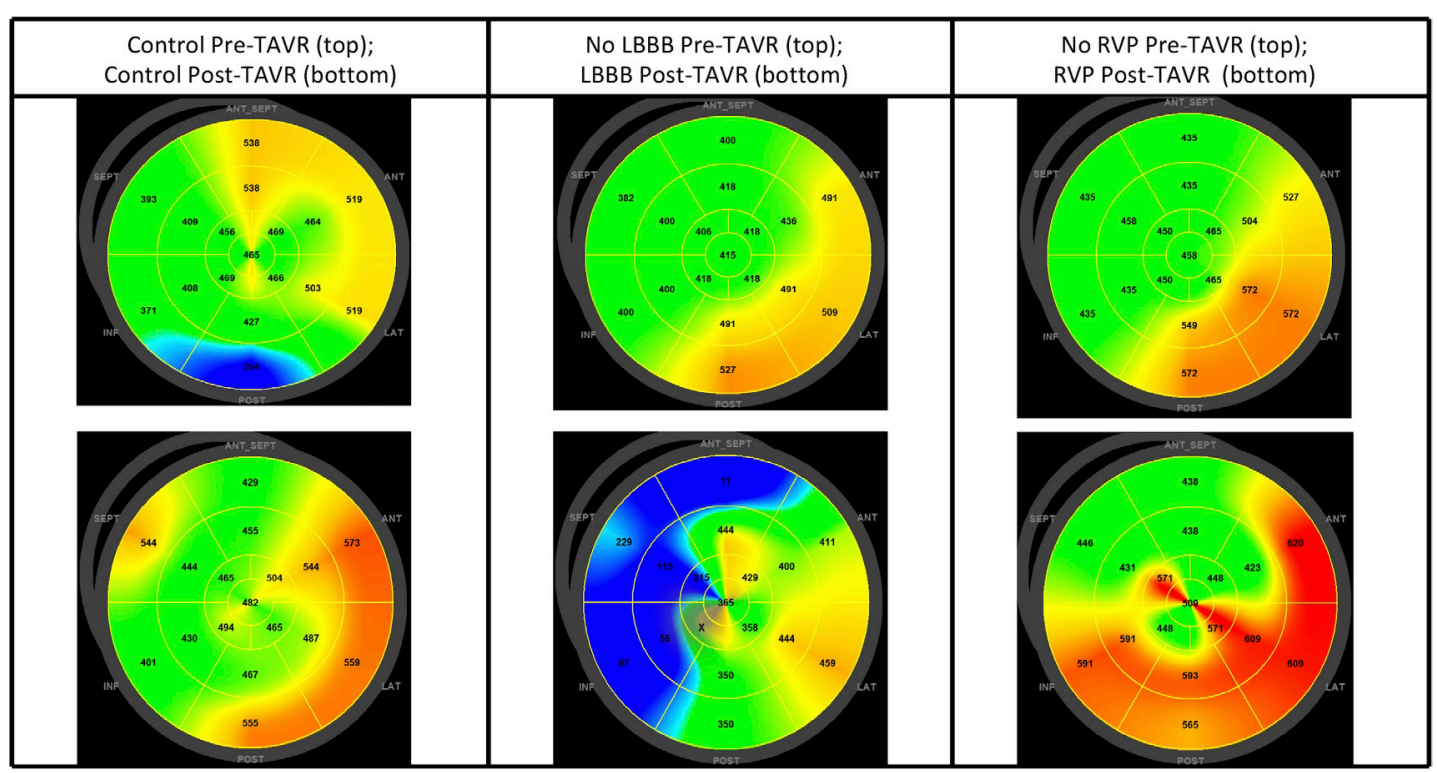

Figure 2 Pre-TAVR and post-TAVR polar maps of time to peak longitudinal strain. Pre-TAVR (top row) and post-TAVR (bottom row) polar maps of time to peak longitudinal strain in representative patients from each group. Left column: a control patient; centre column: a patient who developed LBBB post-TAVR; right column: a patient who required RVP post-TAVR. LBBB, left bundle branch block; RVP, right ventricular paced rhythm; TAVR, transcatheter aortic valve replacement. 


\begin{tabular}{|c|c|c|c|c|c|c|c|c|}
\hline & All patients (r) & $P$ value & Control (r) & $P$ value & LBBB (r) & $P$ value & RVP (r) & $P$ value \\
\hline LVEF and QRS complex duration & -0.3 & 0.1 & -0.4 & 0.3 & -0.2 & 0.5 & -0.2 & 0.7 \\
\hline LVEF and RWH & -0.2 & 0.2 & -0.2 & 0.6 & -0.4 & 0.3 & 0.02 & 0.9 \\
\hline LVEF and TWH & -0.3 & 0.07 & -0.1 & 0.7 & -0.5 & 0.1 & -0.02 & 0.9 \\
\hline GLS and QRS complex duration & -0.2 & 0.2 & -0.3 & 0.3 & -0.3 & 0.4 & -0.1 & 0.7 \\
\hline GLS and RWH & -0.02 & 0.9 & -0.2 & 0.2 & -0.3 & 0.1 & 0.7 & 0.00003 \\
\hline GLS and TWH & -0.1 & 0.4 & -0.2 & 0.2 & -0.3 & 0.1 & 0.03 & 0.8 \\
\hline MD and QRS complex duration & 0.4 & 0.04 & -0.01 & 0.9 & 0.3 & 0.4 & -0.06 & 0.9 \\
\hline $\mathrm{MD}$ and $\mathrm{RWH}$ & 0.1 & 0.01 & -0.02 & 0.9 & 0.4 & 0.01 & -0.3 & 0.09 \\
\hline MD and TWH & 0.4 & 0.1 & -0.02 & 0.9 & 0.7 & 0.00004 & -0.4 & 0.03 \\
\hline
\end{tabular}

GLS, global longitudinal strain; LBBB, left bundle branch block; LVEF, left ventricular ejection fraction; MD, mechanical dispersion; RVP, right ventricular paced rhythm; RWH, R-wave heterogeneity; TAVR, transcatheter aortic valve replacement; TWH, T-wave heterogeneity.

with LVEF. Also, changes in RWH and TWH correlated with GLS and MD but not with LVEF. TAVR-induced LBBBs and RVPs have been inconsistently associated with reduced LVEF, increased heart failure rehospitalisation and increased mortality. ${ }^{3}$ Current guidelines highlight the lack of clinical data regarding follow-up for TAVRinduced LBBBs and the limited data available regarding cardiac resynchronisation therapy in TAVR patients. ${ }^{6}$ RWH, TWH, GLS and MD have been shown to be better predictors of adverse cardiac events than LVEF in various disease states, such as stratifying risk for ventricular arrhythmias and sudden cardiac death independent of LVEF. ${ }^{7-10}$ The correlation among RWH, TWH, GLS and MD in our study suggests that these measures more sensitively identified heterogeneous electromechanical

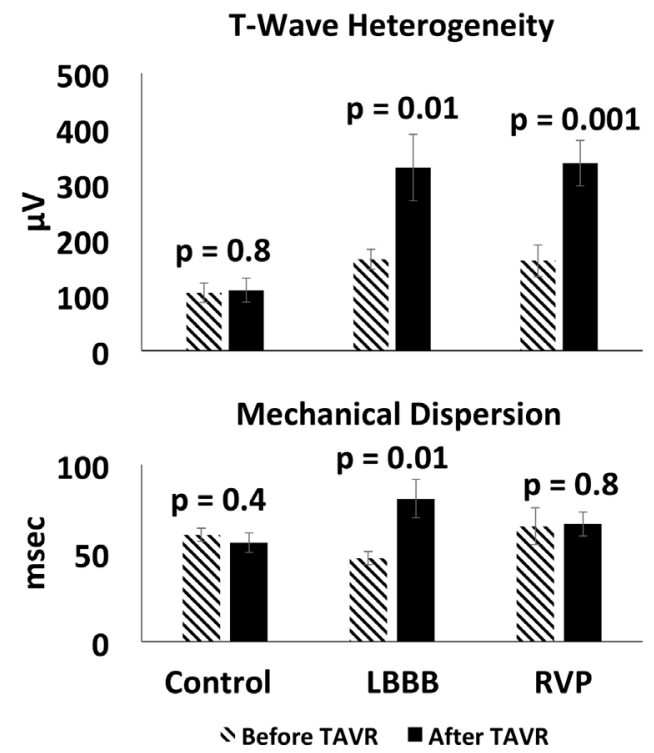

Figure 3 Comparison of changes in T-wave heterogeneity and mechanical dispersion pre-TAVR and post-TAVR. PreTAVR and post-TAVR comparison of changes in T-wave heterogeneity and mechanical dispersion by group. LBBB, left bundle branch block; RVP, right ventricular paced rhythm; TAVR, transcatheter aortic valve replacement. changes than did LVEF. Therefore, these measures may help discriminate among patients with TAVR-induced conduction abnormalities who are at risk for adverse clinical outcomes and assist in guiding post-TAVR clinical management.

\section{Electrocardiographic assessment}

Our results demonstrated prolonged pre-TAVR QRS complex duration and elevated RWH and TWH among patients who developed TAVR-induced LBBBs and RVPs. Further investigation of these findings may allow for more accurate preprocedural risk assessment regarding the risk of developing TAVR-induced LBBBs and RVPs. Also, our results demonstrated increased RWH and TWH post-TAVR in the LBBB and RVP groups. Increased RWH and TWH have been associated with an elevated risk of ventricular arrhythmia and sudden cardiac death. ${ }^{810}$ Baseline RWH and TWH have been shown to predict superresponse (ie, an increase in LVEF of at least 20\%) among patients undergoing cardiac resynchronisation therapy. ${ }^{13}$ Further study of RWH and TWH in patients with TAVRinduced conduction abnormalities may help to identify patients who are at higher risk for adverse events and assist in guiding clinical decision-making regarding indications for permanent pacemaker, cardiac resynchronisation therapy and defibrillator implantation.

\section{Strain assessment}

Left ventricular systolic function on TTE demonstrated a trend toward improvement in the control group, worsening of systolic function in the LBBB group and relative stability in the RVP group, similar to published studies. ${ }^{3}$ GLS and MD have been shown to be better predictors of cardiac outcomes than LVEF in a variety of disease states. Among patients undergoing aortic valve replacement, GLS correlated with severity of left ventricular myocardial fibrosis on biopsy, New York Heart Association class improvement, and mortality whereas LVEF did not. ${ }^{1415}$ Baseline GLS has been shown to be an independent predictor of mortality in patients undergoing cardiac resynchronisation therapy, and MD has been 
shown to be an independent predictor of ventricular arrhythmias 6 months after cardiac resynchronisation therapy implantation. ${ }^{16}$ Impaired GLS and elevated MD have been shown to predict ventricular arrhythmias independent of LVEF and to identify patients at risk for sudden cardiac death who would have benefitted from defibrillator implantation but did not meet current criteria based on LVEF. ${ }^{79}$ Therefore, GLS and MD may offer additional risk stratification pre-TAVR and help to guide post-TAVR clinical decision-making regarding appropriate surveillance and treatment among patients with TAVR-induced conduction abnormalities.

\section{Correlation of electrocardiographic and strain assessments}

Among all patients in our study, MD and QRS complex duration moderately correlated, likely reflecting a similar change in heterogeneous electromechanical left ventricular function. The strong correlation of GLS and RWH in the RVP group could be due to increased left ventricular fibrosis resulting in reduced systolic function and heterogeneous left ventricular electrical activation. MD and TWH in the LBBB group strongly correlated, suggesting a similar identification of heterogeneous myocardial contraction and repolarisation.

In a study evaluating patients with arrhythmogenic right ventricular cardiomyopathy, the risk model that included ECG findings and strain measures yielded the highest accuracy in predicting ventricular arrhythmias. ${ }^{17}$ Therefore, using RWH, TWH, GLS and MD in TAVR patients may assist with identification of patients at risk for TAVR-induced conduction abnormalities, selection of TAVR devices with the best side effect profile for each patient, and guidance of appropriate surveillance and treatments post-TAVR. The current European Society of Cardiology guidelines specifically mention, 'although TAV $[R]$ patients usually meet the criteria for [chronic resynchronization therapy] in patients with conventional indication for anti-bradycardia pacing, there is limited experience of [cardiac resynchronization therapy] in TAV $[R]$ patients'. ${ }^{6}$ In addition to potentially using preTAVR strain imaging and ECG heterogeneity measures to help predict which patients may develop conduction abnormalities as a result of TAVR, the changes in these measures from pre-TAVR to post-TAVR may help to identify which patients are most likely to benefit from cardiac resynchronisation therapy and/or implantation of cardioverter defibrillators.

\section{LIMITATIONS}

Our analysis has several limitations. First, our study was a retrospective, single centre study with a small study population. This small convenience sample did not allow for appropriate age and gender matching as we included all patients with TAVR-induced LBBBs and RVPs who met the ECG and TTE inclusion criteria. The randomly selected control patients (11 of 37) who met the ECG and TTE inclusion criteria had similar baseline clinical characteristics to the larger control cohort but similarly did not allow for appropriate age and gender matching with the other groups. Further study at multiple centres in a prospective fashion should be conducted to determine if these results could be generalised to a larger population. Second, only 58 patients out of the 238 who met the ECG inclusion criteria had pre-TAVR and post-TAVR TTEs that were adequate for strain analysis. Therefore, requirement of adequate image quality may select for certain clinical characteristics, such as body habitus, that could have impacted our results. This relatively low yield of suitable TTEs for strain analysis has been documented in other studies that retrospectively reviewed TTEs that were not originally acquired using a protocol that was optimised for strain analysis. For example, a retrospective study by Tastet and colleagues that evaluated TTE measures as predictors of outcomes among asymptomatic patients with at least moderate aortic stenosis was only able to include strain in one-third of the patients as the TTEs were not originally acquired with the intent to optimise image acquisition and storage for strain analyses. ${ }^{18}$ Additionally, a follow-up study from the Treatment of Preserved Cardiac Function Heart Failure With an Aldosterone Antagonist (TOPCAT) trial that looked at longitudinal strain and outcomes in heart failure with preserved ejection fraction (TOPCAT echo study) demonstrated a low yield of studies eligible for retrospective strain analysis. ${ }^{19}$ The TOPCAT echo study evaluated 935 patients with TTEs from the original TOPCAT trial and ultimately only included 131 patients with baseline and follow-up TTE images adequate for strain analysis. ${ }^{19}$ The authors reported that only $48 \%$ of studies were suitable for strain measurements due to incorrect 'imaging format, missing views, and poor image quality', which mirrors the reasons that limited TTEs for analysis in our study. Further limiting the sample size in the TOPCAT echo study, as well as in our current study, was the need for both baseline and follow-up TTEs to be adequate for strain analysis. Of note, the strain protocol in the TOPCAT echo study used only two apical views (ie, 4 chamber and 2 chamber) to calculate 'longitudinal strain' whereas our study included three apical views (ie, 4 chamber, 2 chamber and 3 chamber) to calculate GLS and MD, demonstrating a relatively favourable yield of adequate TTEs in our study. ${ }^{19}$ Third, the scope of our study is limited to findings on TTE and ECG as we did not include adverse events or mortality.

\section{CONCLUSION}

In this convenience sample, our findings demonstrate that QRS complex duration, RWH and TWH correlate more strongly with GLS and MD than LVEF in patients with TAVR-induced LBBBs and RVPs. Our results are consistent with prior studies showing that GLS, MD, RWH and TWH are able to detect subclinical changes in cardiac function prior to developing changes in LVEF. Further investigation using GLS, MD, RWH and TWH may offer 
additional insights into pre-TAVR risk assessment and post-TAVR surveillance and treatment in patients with TAVR-induced conduction abnormalities such as timing of and indications for implantation of permanent pacemakers, implantation of or upgrade to cardiac resynchronisation therapy, and timing of and indications for implantation of cardioverter defibrillators.

Contributors GMB is responsible for the overall content as guarantor. GMB developed the study design, screened medical records for inclusion in the study, interpreted ECGs, interpreted transthoracic echocardiograms, and was the primary writer of the manuscript. BAS, AAM and ALB worked under the mentorship of RLV, contributed to the study design, conducted electrocardiography measures and contributed to writing the manuscript. BDN was primarily responsible for the statistical analyses performed in this study. He and RLV are coinventors of the second central moment analysis to quantify R-wave and T-wave heterogeneity. MJC assisted with the IRB approval process and organised demographic and clinical data for patients included in the study. SF assisted in advanced echocardiographic measurements and secure storage of ECGs and imagingrelated measurements. JJP was one of the primary interventional cardiologists at Beth Israel Deaconess Medical who performed the transcatheter aortic valve replacements. Under a protocol approved by the Institutional Review Board of Beth Israel Deaconess Medical Center, he provided access and information regarding patients who underwent this procedure at Beth Israel Deaconess Medical Center. RLV provided expertise in the field of electrocardiography, contributed to the study design, and oversaw electrocardiography measurements including R-wave heterogeneity and T-wave heterogeneity. JDC developed the initial concept for the study and provided mentorship and expertise in speckle tracking strain.

Funding The authors have not declared a specific grant for this research from any funding agency in the public, commercial or not-for-profit sectors.

Competing interests JJP is employed by Medtronic (Minneapolis, MN) and receives research grants and personal fees from Edwards Lifesciences (Irvine, CA), grants from Boston Scientific (Marlborough, MA) and grants from Abbott Vascular (Murrieta, CA). RLV and BDN are investigators in research grants from LivaNova USA (Houston, TX), Medtronic (Minneapolis, MN), Preventice Solutions (Houston, TX)/Boston Scientific (Marlborough, MA) and StratusNeuro (Irving, TX) to Beth Israel Deaconess Medical Center. BAS, ALB and AAM received scholarship support from the Lemann Foundation (São Paulo, Brazil). None of the other authors declares any conflict of interest.

Patient consent for publication Not required.

Ethics approval The Institutional Review Board at Beth Israel Deaconess Medical Center (Boston, Massachusetts, USA) approved our study protocol.

Provenance and peer review Not commissioned; externally peer reviewed.

Data availability statement Data are available upon reasonable request. All patient data were stored in compliance with the Beth Israel Deaconess Medical Center IRB protocol. Requests for data will need to be processed through the Beth Israel Deaconess Medical Committee on Clinical Investigations to obtain access.

Open access This is an open access article distributed in accordance with the Creative Commons Attribution Non Commercial (CC BY-NC 4.0) license, which permits others to distribute, remix, adapt, build upon this work non-commercially, and license their derivative works on different terms, provided the original work is properly cited, appropriate credit is given, any changes made indicated, and the use is non-commercial. See: http://creativecommons.org/licenses/by-nc/4.0/.

ORCID iD

Gordon M Burke http://orcid.org/0000-0002-4724-6548

\section{REFERENCES}

1 Popma JJ, Deeb GM, Yakubov SJ, et al. Transcatheter aortic-valve replacement with a self-expanding valve in low-risk patients. $N$ Engl J Med 2019;380:1706-15.
2 Mack MJ, Leon MB, Thourani VH, et al. Transcatheter aortic-valve replacement with a balloon-expandable valve in low-risk patients. $N$ Engl J Med 2019;380:1695-705.

3 Auffret V, Puri R, Urena M, et al. Conduction disturbances after transcatheter aortic valve replacement: current status and future perspectives. Circulation 2017;136:1049-69.

4 Vassallo JA, Cassidy DM, Miller JM, et al. Left ventricular endocardial activation during right ventricular pacing: effect of underlying heart disease. J Am Coll Cardiol 1986;7:1228-33.

5 Vassallo JA, Cassidy DM, Marchlinski FE, et al. Endocardial activation of left bundle branch block. Circulation 1984;69:914-23.

6 European Society of Cardiology (ESC), European Heart Rhythm Association (EHRA), Brignole M, et al. 2013 ESC guidelines on cardiac pacing and cardiac resynchronization therapy: the task force on cardiac pacing and resynchronization therapy of the European Society of cardiology (ESC). developed in collaboration with the European heart rhythm association (EHRA). Europace 2013;15:1070-118.

7 Haugaa KH, Grenne BL, Eek CH, et al. Strain echocardiography improves risk prediction of ventricular arrhythmias after myocardial infarction. JACC Cardiovasc Imaging 2013;6:841-50.

8 Tan AY, Nearing BD, Rosenberg M, et al. Interlead heterogeneity of R- and T-wave morphology in standard 12-lead ECGs predicts sustained ventricular tachycardia/fibrillation and arrhythmic death in patients with cardiomyopathy. $J$ Cardiovasc Electrophysiol 2017;28:1324-33.

9 Perry R, Patil S, Marx C, et al. Advanced echocardiographic imaging for prediction of SCD in moderate and severe LV systolic function. JACC Cardiovasc Imaging 2020;13:604-12.

10 Kenttä TV, Nearing BD, Porthan K, et al. Prediction of sudden cardiac death with automated high-throughput analysis of heterogeneity in standard resting 12-lead electrocardiograms. Heart Rhythm 2016;13:713-20.

11 Surawicz B, Childers R, Deal BJ, et al. AHA/ACCF/HRS recommendations for the standardization and interpretation of the electrocardiogram: Part III: intraventricular conduction disturbances: a scientific statement from the American heart association electrocardiography and arrhythmias Committee, Council on clinical cardiology; the American College of cardiology Foundation; and the heart rhythm Society. endorsed by the International Society for computerized Electrocardiology. J Am Coll Cardiol 2009;53:976-81.

12 Voigt J-U, Pedrizzetti G, Lysyansky P, et al. Definitions for a common standard for 2D speckle tracking echocardiography: consensus document of the EACVI/ASE/Industry Task force to standardize deformation imaging. J Am Soc Echocardiogr 2015;28:183-93.

13 Bortolotto AL, Verrier RL, Nearing BD, et al. Preimplantation interlead ECG heterogeneity is superior to QRS complex duration in predicting mechanical super-response in patients with non-left bundle branch block receiving cardiac resynchronization therapy. Heart Rhythm 2020;17:1887-96.

14 Park S-J, Cho SW, Kim SM, et al. Assessment of Myocardial Fibrosis Using Multimodality Imaging in Severe Aortic Stenosis: Comparison With Histologic Fibrosis. JACC Cardiovasc Imaging 2019;12:109-19.

15 Kempny A, Diller G-P, Kaleschke G, et al. Longitudinal left ventricular $2 \mathrm{D}$ strain is superior to ejection fraction in predicting myocardial recovery and symptomatic improvement after aortic valve implantation. Int J Cardiol 2013;167:2239-43.

16 Hasselberg NE, Haugaa KH, Bernard A, et al. Left ventricular markers of mortality and ventricular arrhythmias in heart failure patients with cardiac resynchronization therapy. Eur Heart $J$ Cardiovasc Imaging 2016;17:343-50.

17 Lie Øyvind H, Rootwelt-Norberg C, Dejgaard LA, et al. Prediction of life-threatening ventricular arrhythmia in patients with arrhythmogenic cardiomyopathy: a primary prevention cohort study. JACC Cardiovasc Imaging 2018;11:1377-86.

18 Tastet L, Tribouilloy C, Maréchaux S, et al. Staging cardiac damage in patients with asymptomatic aortic valve stenosis. J Am Coll Cardiol 2019;74:550-63.

19 Shah AM, Claggett B, Sweitzer NK, et al. Prognostic importance of impaired systolic function in heart failure with preserved ejection fraction and the impact of spironolactone. Circulation 2015;132:402-14. 